\title{
Doses of intensity-modulated radiotherapy and its association with cardiac disease in esophageal cancer patients
}

\author{
Jiandong Zhang ${ }^{1}$, Yajuan $\mathrm{Lv}^{2}$, Fangjie Chen ${ }^{3}$, Xiaotong Wang ${ }^{4}$, Li Zhang ${ }^{2}$, Xiaozhi Zhang ${ }^{1}$ \\ ${ }^{1}$ Department of Radiation Oncology, The First Affiliated Hospital of Xi'an Jiaotong University, Xi'an, China; ${ }^{2}$ Department of Oncology, The First \\ Affiliated Hospital of Shandong First Medical University \& Shandong Provincial Qianfoshan Hospital, Jinan, China; ${ }^{3}$ Department of Oncology, \\ Renji Hospital Affiliated with Shanghai Jiao Tong University, Shanghai, China; ${ }^{4}$ Shandong First Medical University, Jinan, China \\ Contributions: (I) Conception and design: J Zhang, X Zhang; (II) Administrative support: X Zhang; (III) Provision of study materials or patients: F \\ Chen; (IV) Collection and assembly of data: Y Lv; (V) Data analysis and interpretation: X Wang, L Zhang; (VI) Manuscript writing: All authors; (VII) \\ Final approval of manuscript: All authors. \\ Correspondence to: Xiaozhi Zhang. Department of Radiation Oncology, The First Affiliated Hospital of Xi'an Jiaotong University, 277 West Yanta \\ Road, Xi'an 700617, China. Email: zhang9149@sina.com.
}

Background: No clear guidelines or available studies exist regarding the effects of intensity-modulated radiotherapy (IMRT) of esophageal cancer (EC) on the cardiovascular system. We therefore analyzed a wide range of cardiac vascular dosimetric parameters and clinical characteristics to assess the prognostic factors for EC patients treated with IMRT.

Methods: A total of 112 patients receiving IMRT at the Qianfoshan Hospital between July 2012 and May 2017 were retrospectively reviewed. The dose per fraction was 1.8-2.0 Gy, and the total dose range was 5466 Gy. Kaplan-Meier analysis was used to estimate death due to heart disease. Univariate and multivariate logistic regression models were calculated to test for associations between patient characteristics and dosevolume histogram (DVH) parameters. A $t$-test and chi-squared or Fisher's exact test was used to analyze the comparisons.

Results: The maximum and mean doses received by the heart were 57.34 \pm 13.51 and $24.83 \pm 11.40$ Gy, respectively. Among the parameters examined, which included the maximum dose received by the heart, the mean dose received by the right and left ventricle (RV and LV), and the maximum dose received by the right atrium (RA), the mean dose received by the RV predicted survival and was included in our multivariate analysis. The results indicated that patients with basic heart disease who were undergoing concurrent radiochemotherapy were more likely to have cardiac disease.

Conclusions: This is first study to examine the prognosis of cardiovascular vessels exposed to various radiation doses during the treatment of $\mathrm{EC}$, the findings of which suggest that limiting radiation exposure may be an important measure in IMRT application. These findings of this study may provide theoretical support for prediction of radiation-induced heart disease (RIHD). Furthermore, to curb the risk of RIHD, the modality of chemotherapy also needs to be attentively monitored and managed.

Keywords: Cardiovascular radiation doses; radiation-induced heart disease (RIHD); intensity-modulated radiotherapy (IMRT); prognosis; esophageal cancer (EC)

Submitted Dec 10, 2020. Accepted for publication Jan 22, 2021.

doi: $10.21037 / \mathrm{atm}-21-184$

View this article at: http://dx.doi.org/10.21037/atm-21-184 


\section{Introduction}

The incidence of esophageal cancer (EC) is rapidly increasing compared to that of other malignancies, and EC is now the sixth leading cause of tumor mortality (1). In the treatment of EC, radiotherapy (RT) is a major modality for unresectable disease, but preoperative RT can contribute to operable disease as well (2). Intensity-modulated radiotherapy (IMRT) can be integral to reducing toxicity and sparing normal tissue (3). Radiation to the adjacent tissue typically occurs to the heart, which is a late-reacting organ. Radiation has been implicated in increasing the risk of cardiac toxicity in breast cancer and Hodgkin's disease $(4,5)$, and correlated factors have been identified, including the heart volume, total amount of radiation, and fraction size (6-9). However, the clinical awareness of radiationassociated cardiovascular toxicity in esophageal carcinoma is unsatisfactorily low.

Cardiovascular insults resulting from radiation exposure usually manifest as a long latent period with subclinical changes, including coronary artery disease (CAD), ischemia, myocardial fibrosis, and valvular insufficiency with pericardial disease $(10,11)$. There is a paucity of data regarding the cardiac complications resulting from the radiation treatment of $\mathrm{EC}$, mainly due to the poor longterm survival of these patients. The articles that do exist report conflicting findings concerning the relationship between the radiation dose to the heart and the associated cardiac toxicity (11).

To our knowledge, no clear guidelines or available published studies currently exist regarding the prognosis of the cardiovascular system after patients are treated with IMRT for EC. In this study, we retrospectively analyzed a wide range of cardiac vascular dosimetric parameters to assess the prognostic factors for EC patients treated with IMRT. This is first study to examine the prognosis of cardiovascular vessels exposed to various radiation doses during the treatment of EC, the findings of which suggest that limiting radiation exposure may be an important measure in IMRT application. These findings of this study may provide theoretical support for prediction of radiationinduced heart disease. Our study showed the correlations between the radiation dose to the cardiac substructure and RIHD has not been reported in the past. We present the following article in accordance with the STROBE reporting checklist (available at http://dx.doi.org/10.21037/ atm-21-184).

\section{Methods}

\section{Patients}

Between July 2012 and May 2017, the medical records of 112 patients receiving RT for EC at the Department of Radiation Oncology, Qianfoshan Hospital, were retrospectively reviewed following institutional review board approval. The evaluated patients had biopsyconfirmed clinical stage II-IV esophageal carcinoma that was managed with RT. To avoid underestimating the incidence of cardiac disease, we identified patients with a follow-up period of $\geq 60$ months. Reasons for ineligibility included a previously treated malignancy and a history of thoracic radiation. All patients who participated in this study provided written informed consent for publication. This study was approved by the Board and Ethical Committee of Qianfoshan Hospital and was conducted in accordance with the Declaration of Helsinki (as revised in 2013).

\section{Treatment}

RT was administered with $6 \mathrm{MV}$ photons 5 days per week. The doses per fraction were 1.8-2.0 Gy, and the total dose ranged from 56 to $60 \mathrm{~Gy}$. The IMRT regimen was performed in all radiation treatment plans. None of the patients received further radiation in addition to the session included in the initial planned course. During this time, computed tomography (CT) scans using intravenous and oral contrast agents were obtained for all patients. Images were acquired with the participants in the supine position. All procedures were performed with the Eclipse 10.0 Treatment Planning System (Varian Medical Systems Inc., Palo Alto, CA, USA).

The gross tumor volume (GTV) was defined as the region of the primary tumor along with any related lymph nodes ( $>1 \mathrm{~cm}$ on the short axis) and was determined by the treating radiation oncologist. A $1.5-\mathrm{cm}$ circumferential and 4-cm superior/inferior expansion of this area, including the involved contours of the GTV, was defined as the clinical target volume (CTV). After considering the daily setup error and motion, the planning target volume (PTV) was expanded by $0.5 \mathrm{~cm}$ in the radial dimension beyond the $\mathrm{CTV}$ to address these issues.

\section{Dosimetric analysis}

Two physicians contoured the whole external heart and 
coronary border, while another radiation oncologist examined this area. Dose-volume histograms (DVHs) of the PTV, heart, and critical normal heart arteries were generated, and the risks to other organs (lungs, stomach, liver, and spinal cord) were calculated using the treatment planning system. The volumes of the heart's right coronary artery (RCA), left anterior descending artery (LAD), left circumflex artery (LCX), left ventricle (LV), left atrium (LA), right ventricle (RV), and right atrium (RA) were recorded for each patient.

\section{Statistical analyses}

All statistical analyses were performed with Statistical Package for the Social Sciences, version 21.0 (IBM Corp., Armonk, NY, USA). We employed standard descriptive statistics, including two-sided $95 \%$ confidence intervals (CI), to analyze the study population. Kaplan-Meier analysis was used to estimate death due to heart disease, which was considered the primary endpoint in the enrolled patients. The percentage of heart artery volume and the maximum and mean doses received by the heart were calculated from the DVHs. Receiver operating characteristic (ROC) curve analysis was used to determine the cutoff values for the DVH parameters. Furthermore, the areas under the ROC curves were calculated to assess the discriminative power of the models. Univariate and multivariate logistic regression models were used to test for associations between patient characteristics and DVH parameters. The explanatory variables were separately controlled to evaluate cardiac survival. A $t$-test and Chi-squared or Fisher's exact test were used to analyze the comparisons. A two-tailed $\mathrm{P}$ value less than 0.05 was defined as statistically significant.

\section{Results}

\section{Patient and tumor characteristics}

A summary of the baseline characteristics of the $112 \mathrm{EC}$ patients treated with an RT modality is listed in Table 1. Among the patients, the median age was 67 years, and the age range was 49 to 85 years. In this cohort, patients were predominantly male (84\%), while only $16 \%$ were female. Approximately $76 \%$ of patients had squamous cell carcinoma, and $24 \%$ had adenocarcinoma; 40 patients had stage II, 52 patients had stage III, and 20 had stage IV disease according to the database. The majority of patients did not have basic heart disease before treatment. The
Table 1 Patient and tumor characteristics

\begin{tabular}{|c|c|c|}
\hline Characteristic & No. & $\%$ \\
\hline \multicolumn{3}{|l|}{ Age (years) } \\
\hline$\leq 65$ & 48 & 43 \\
\hline$>65$ & 64 & 57 \\
\hline \multicolumn{3}{|l|}{ Sex } \\
\hline Male & 95 & 84 \\
\hline Female & 17 & 16 \\
\hline \multicolumn{3}{|l|}{ Stage } \\
\hline II & 40 & 36 \\
\hline III & 52 & 46 \\
\hline IV & 20 & 18 \\
\hline \multicolumn{3}{|l|}{ Histology } \\
\hline Squamous & 85 & 76 \\
\hline Adenocarcinoma & 27 & 24 \\
\hline \multicolumn{3}{|l|}{ Basic heart disease } \\
\hline Yes & 29 & 26 \\
\hline No & 83 & 74 \\
\hline \multicolumn{3}{|l|}{ RIHD } \\
\hline Yes & 54 & 48 \\
\hline No & 58 & 52 \\
\hline \multicolumn{3}{|c|}{ Concurrent chemotherapy } \\
\hline Yes & 66 & 59 \\
\hline No & 46 & 41 \\
\hline \multicolumn{3}{|c|}{ Consolidation chemotherapy } \\
\hline Yes & 38 & 34 \\
\hline No & 74 & 66 \\
\hline
\end{tabular}

RIHD, radiation-induced heart disease.

patients with and without radiation-induced heart disease (RIHD) were approximately equal in all groups. In terms of modality, 66 patients were treated with concurrent chemoradiotherapy, and a total of 38 patients had a history of consolidation chemotherapy after RT.

\section{DVH parameters}

Patient descriptive dosimetric characteristics are presented in Table 2. Data are presented as the mean \pm standard deviation (SD). The maximum (Dmax) and mean doses 
Table 2 Doses in the target volume and heart for all patients undergoing IMRT

\begin{tabular}{lcc}
\hline Site & Parameter & IMRT (mean \pm SD) \\
\hline Heart & Dmax (Gy) & $57.34 \pm 13.51$ \\
RA & Dmean (Gy) & $24.83 \pm 11.40$ \\
& Dmax (Gy) & $40.98 \pm 22.64$ \\
RV & Dmean (Gy) & $18.56 \pm 12.87$ \\
& Dmax (Gy) & $36.61 \pm 22.11$ \\
RCA & Dmean (Gy) & $16.47 \pm 13.14$ \\
& Dmax (Gy) & $26.81 \pm 19.50$ \\
LA & Dmean (Gy) & $22.24 \pm 16.58$ \\
& Dmax (Gy) & $46.62 \pm 22.48$ \\
LV & Dmean (Gy) & $35.17 \pm 20.43$ \\
& Dmax (Gy) & $37.08 \pm 22.90$ \\
LCX & Dmean (Gy) & $12.61 \pm 10.84$ \\
& Dmax (Gy) & $31.43 \pm 23.37$ \\
LAD & Dmean (Gy) & $22.66 \pm 19.11$ \\
& Dmax (Gy) & $21.18 \pm 14.68$ \\
\hline
\end{tabular}

IMRT, intensity-modulated radiotherapy; Dmax, maximum dose; Dmean, mean dose; SD, standard deviation; RA, right atrium; $\mathrm{RV}$, right ventricle; RCA, right coronary artery; LA, left atrium; LV, left ventricle; LCX, left circumflex artery; LAD, left anterior descending artery.

received by the heart were $57.34 \pm 13.51$ and $24.83 \pm 11.40 \mathrm{~Gy}$, respectively. An overview of the RT process is summarized in Figure 1.

\section{Univariate and multivariate analysis}

Univariate analyses of patient demographics and heart dosimetric volumes are provided in Table 3. The Dmax of the heart, RA, RV, RCA, LA, LV, and LAD; the mean dose received by the heart; and the RCA, LA, and LAD dosimetric volumes were significantly different and affected cardiac toxicity. The Dmax and mean doses received by the RV and LV and the Dmax of the RA, RV, DVH were predictive of survival and thus were included in our multivariate analysis.

\section{Association between RIHD and survival}

The chi-squared test was used to compare patient variables with RIHD, which demonstrated that patients with both basic heart disease and concurrent radiochemotherapy were more likely to experience cardiac disease. The remaining factors, including age, sex, tumor stage, pathology, and consolidation chemotherapy were not correlated with the incidence of heart events and are described in detail in Table 4.

In the Kaplan-Meier analysis (Figure 2), aside from the mean dose received by the RA, RV, and $\mathrm{LV}$, the remaining dosimetric factors exhibited significant differences in patient survival (all $\mathrm{P}<0.05$ ).

\section{Discussion}

$\mathrm{RT}$ is an essential component of EC management. The heart of EC patients is affected by the irradiation, leading to myocardial fibrosis, coronary artery disease, and valvular lesions, which are considered radiation-induced heart diseases. Delayed cardiac injury especially myocardial fibrosis is prominent, and its incidence is as high as 20 $80 \%$. Myocardial fibrosis is the final stage of radiationinduced heart diseases, and it increases the stiffness of the myocardium and decreases myocardial systolic and diastolic function, resulting in myocardial electrical physiological disorder, incomplete heart function, or even death (12). The exact mechanism causing the occurrence of RIHD is unclear, the possible mechanism is direct damage from radiation is the most important cause. Rays can directly cause tissue ionization, cause local aseptic inflammation, and inhibit the growth of heart cells, causing cell lysis, apoptosis, and even necrosis. Repeated radiation damage can inhibit fibrosis around myocardial cells, so a large amount of cellulose deposited in the cells cannot be discharged, resulting in damage to the vascular endothelium, resulting in changes in vascular permeability, microthrombosis in the blood vessels and reduced blood flow. In addition, the biological effects caused by radiation may cause secondary damage to the heart, such as autoimmune changes in tissues and cells caused by radiation, gene mutations or abnormal gene expression, and obstruction of capillary and lymphatic return. These can aggravate or initiate continuous myocardial damage, accelerate myocardial fibrosis, and aggravate myocardial and pericardial exudation and thickening changes (12). For instance, one study reported that patients receiving RT treatment were 1.62 times more likely to die from heart disease than those without RT (13). Other researchers have reported that IMRT can mitigate the risk of cardiac sequelae (14). In addition, Beukema et al. 

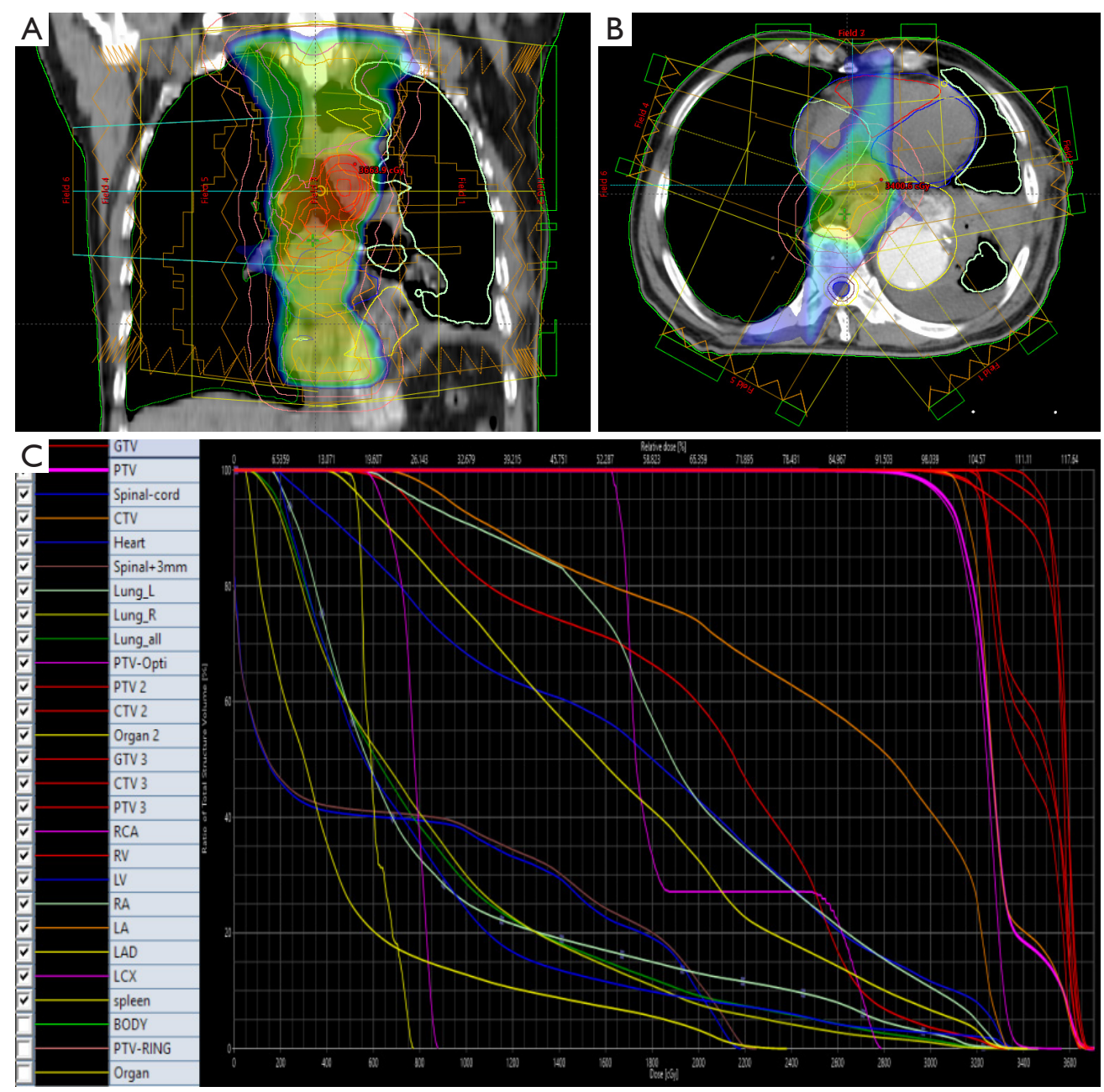

Figure 1 Treatment planning example: an image of a patient treated with radiation. (A,B) Dose distribution image; (C) dose-volume histograms showing the GTV (gold), CTV (dark blue), PTV (magenta), heart (green), lung-all (aqua), LA (orange), RCA (gray), RV (red), LV (blue), RA (light blue), LAD (pink), LCX (white), and spinal cord (yellow) in intensity-modulated radiotherapy (IMRT) treatment plans. GTV, gross tumor volume; CTV, clinical target volume; PTV, planning target volume; LA, left atrium; RCA, right coronary artery; RV, right ventricle; LV, left ventricle; LAD, left anterior descending artery; LAD, left anterior descending artery; LCX, left circumflex artery.

stated that further cardiac function parameters during follow-up were needed to identify the most important parts of the heart (15). Minimizing radiation exposure in normal tissue structures, especially the heart and coronary arteries, and increasing the long-term survival of EC patients is challenging. One of the strengths of our research that addresses this issue is the inclusion of a wide range of heart artery doses in our analysis. To the best of our knowledge, this is the first study to specifically investigate various heart artery radiation doses.

In our population-based analysis of EC survivors, accurate multivariable prediction models of radiation- induced toxicity showed that the Dmax and mean doses in the LV and RV and the Dmax in the RA and RV were significantly different. We determined the cutoff doses for the endpoints, and the risk of death increased with the increase of dosage.

An overview of the relevant randomized trials showed that RT contributed to reducing breast cancer mortality, but also increased cardiovascular mortality (16). In a retrospective study of 415 patients with Hodgkin's disease treated with RT. Hull et al. found that CAD occurred within 5 to 20 years (7). Furthermore, Beukema et al. found that modern RT contributed to increasing the morbidity 
Table 3 Univariable and multivariable Cox regression analysis of treatment and dosimetric characteristics in patients

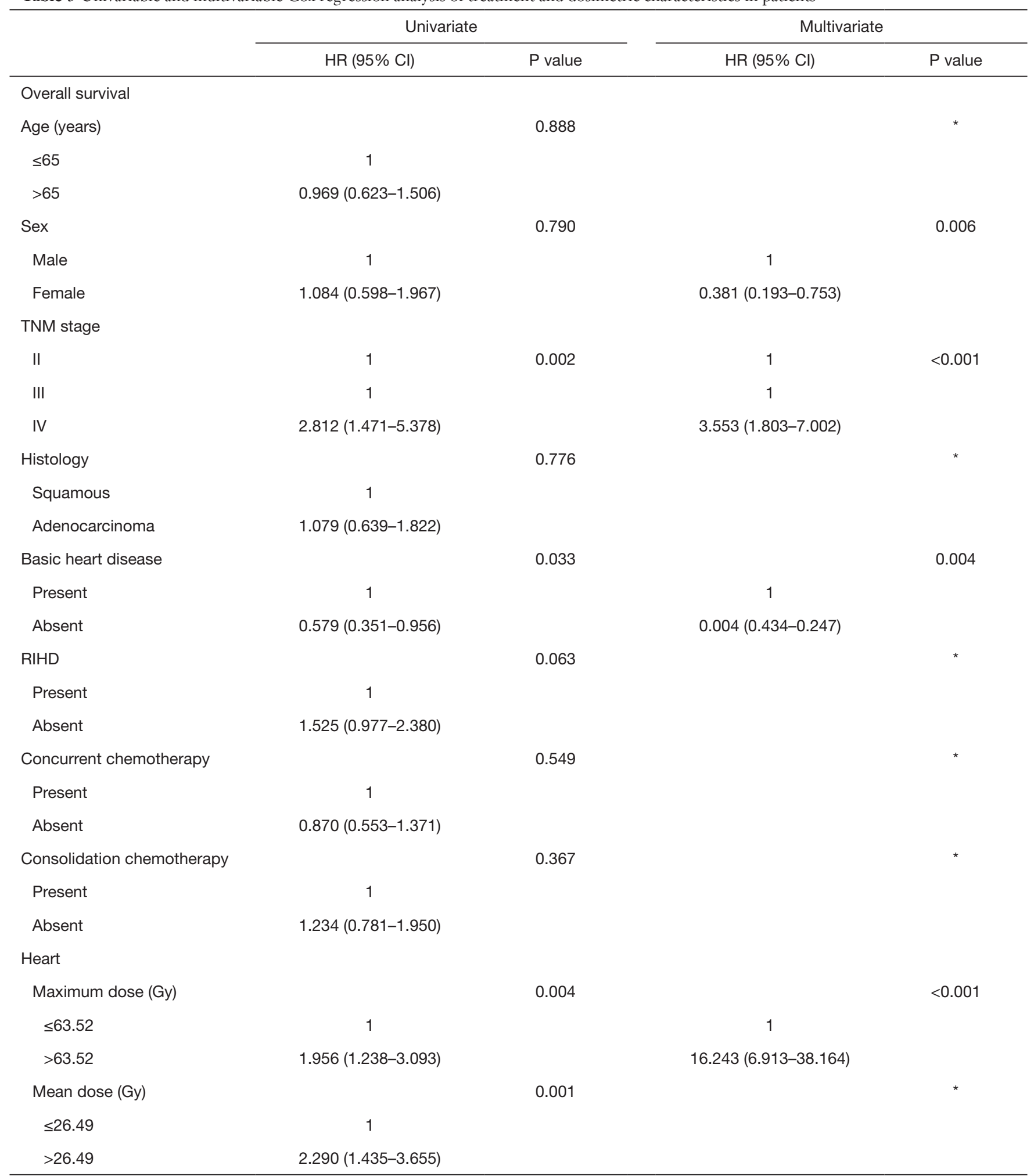

Table 3 (continued) 
Table 3 (continued)

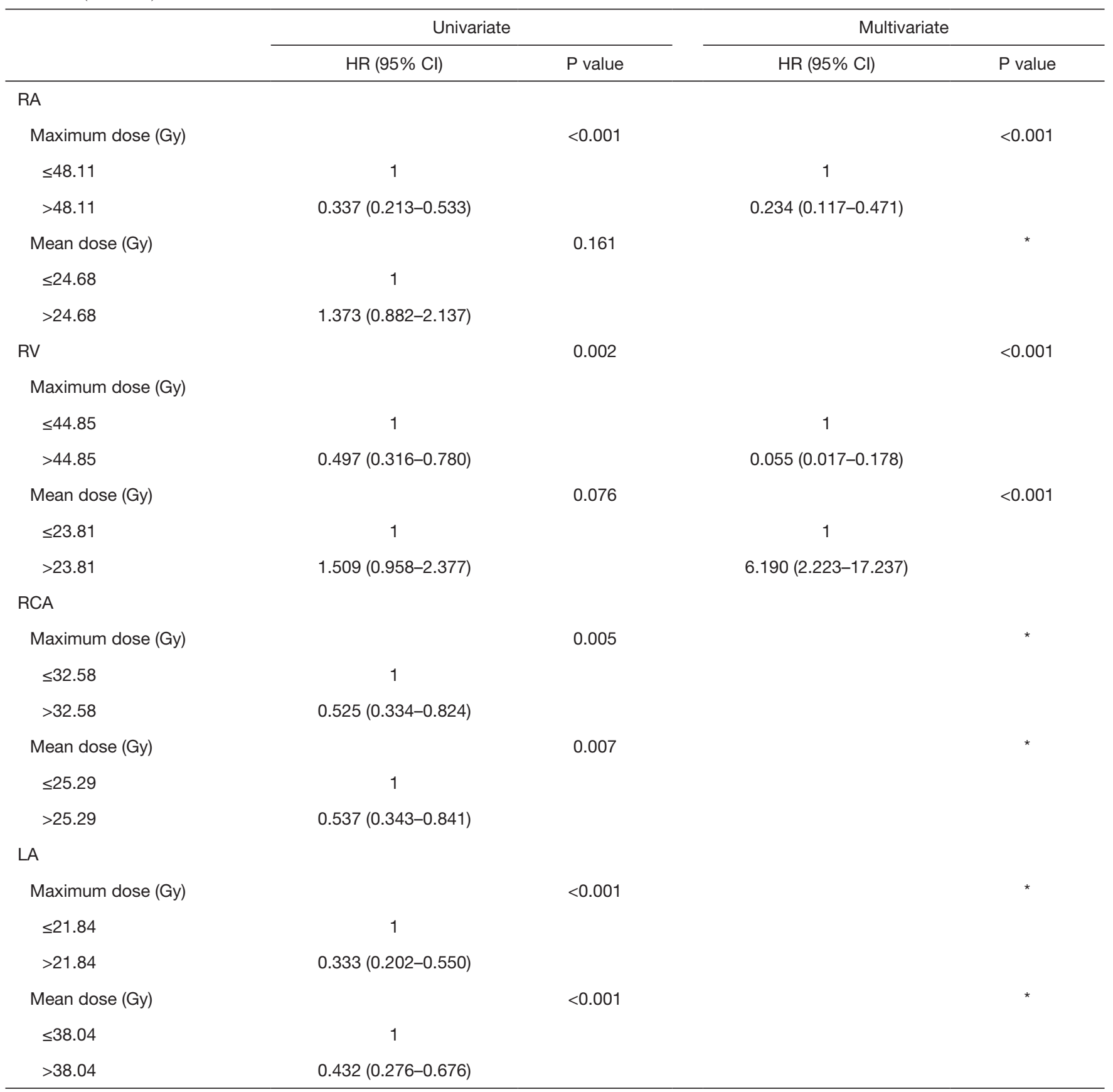

Table 3 (continued) 
Table 3 (continued)

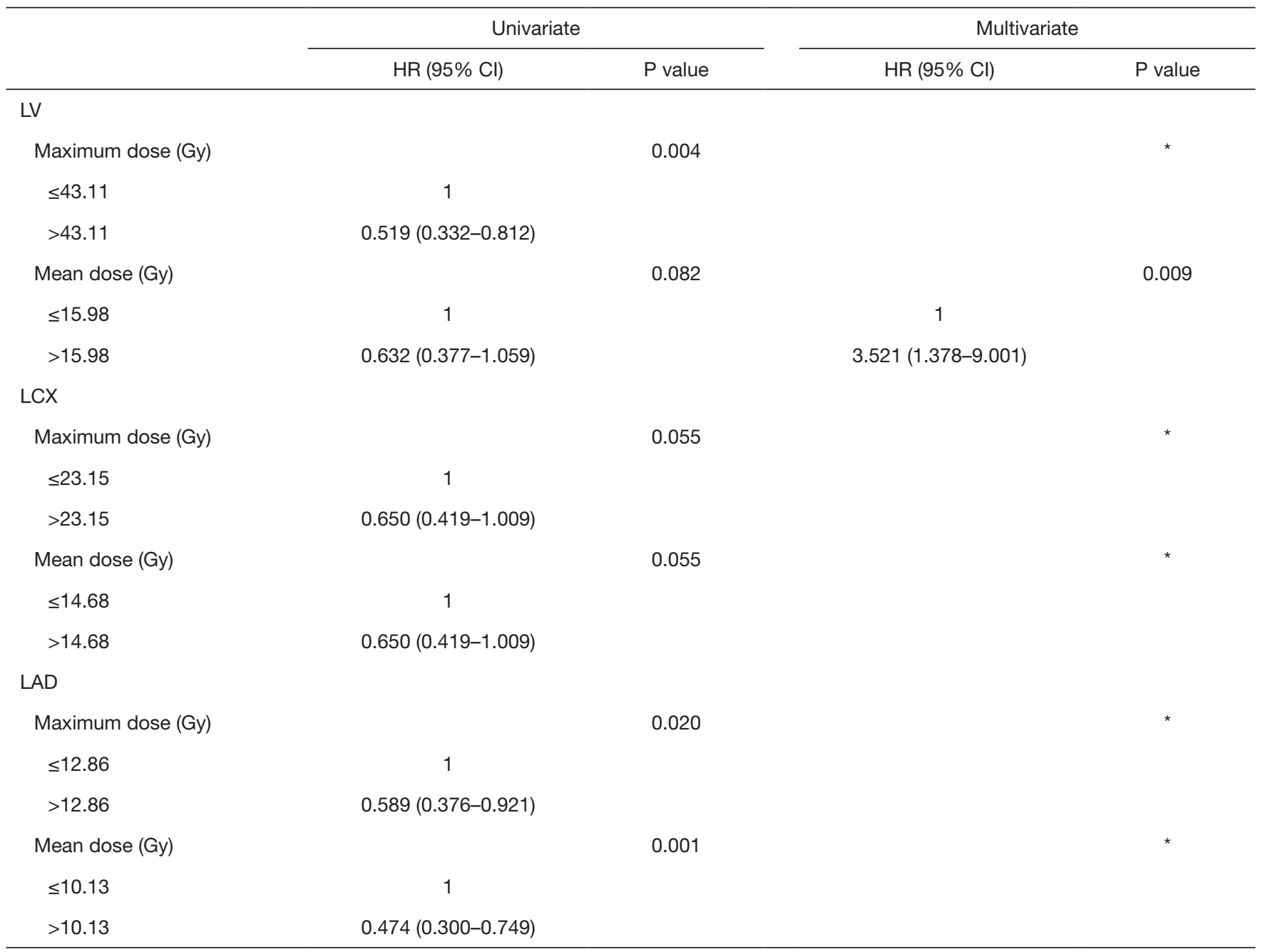

* indicates a variation not in the model. RIHD, radiation-induced heart disease; HR, hazard ratio; $95 \% \mathrm{Cl}$, 95\% confidence interval; TNM, tumor-node-metastasis; RA, right atrium; RV, right ventricle; RCA, right coronary artery; LA, left atrium; LV, left ventricle; LCX, left circumflex artery; LAD, left anterior descending artery.

and mortality of EC due to CAD (15). Previous experience has shown that epicardial CAD is the deadliest complication after radiation but rarely occurs and is treatable (17). In addition, management of radiation-induced $\mathrm{CAD}$, which is commonly included in RIHD, appears similar to the that of normal CAD. These findings led us to examine the arteries of the heart after radiation treatment. Although many RIHD studies of Hodgkin's disease and breast cancer exist, reports of RIHD after radiation for EC are lacking, and the risk factors are unclear. It is possible that the prognosis of EC is worse and the heart radiation doses are higher, resulting in the differences among EC, breast cancer, and lymphoma patients. We searched the database and compiled information regarding heart radiation doses and RIHD. Notably, Lorenzen et al. showed that, compared with the dose received by the LAD coronary artery, the mean dose received by the heart had increased risk of ischemic events; however, they did mention that further confirmation of this findings was needed through further study (18).

Several decades ago, Gyenes et al. showed that cardiac mortality occurs in a dose-volume-dependent manner by demonstrating higher incidences in a highest dosevolume study group than in a low dose-volume group (19). More recently, researchers have attempted to quantify the survival of EC patients according to heart radiation dose and cardiac physiological results. Moreover, Frandsen et 
Table 4 Relationships between RIHD and clinical characteristics

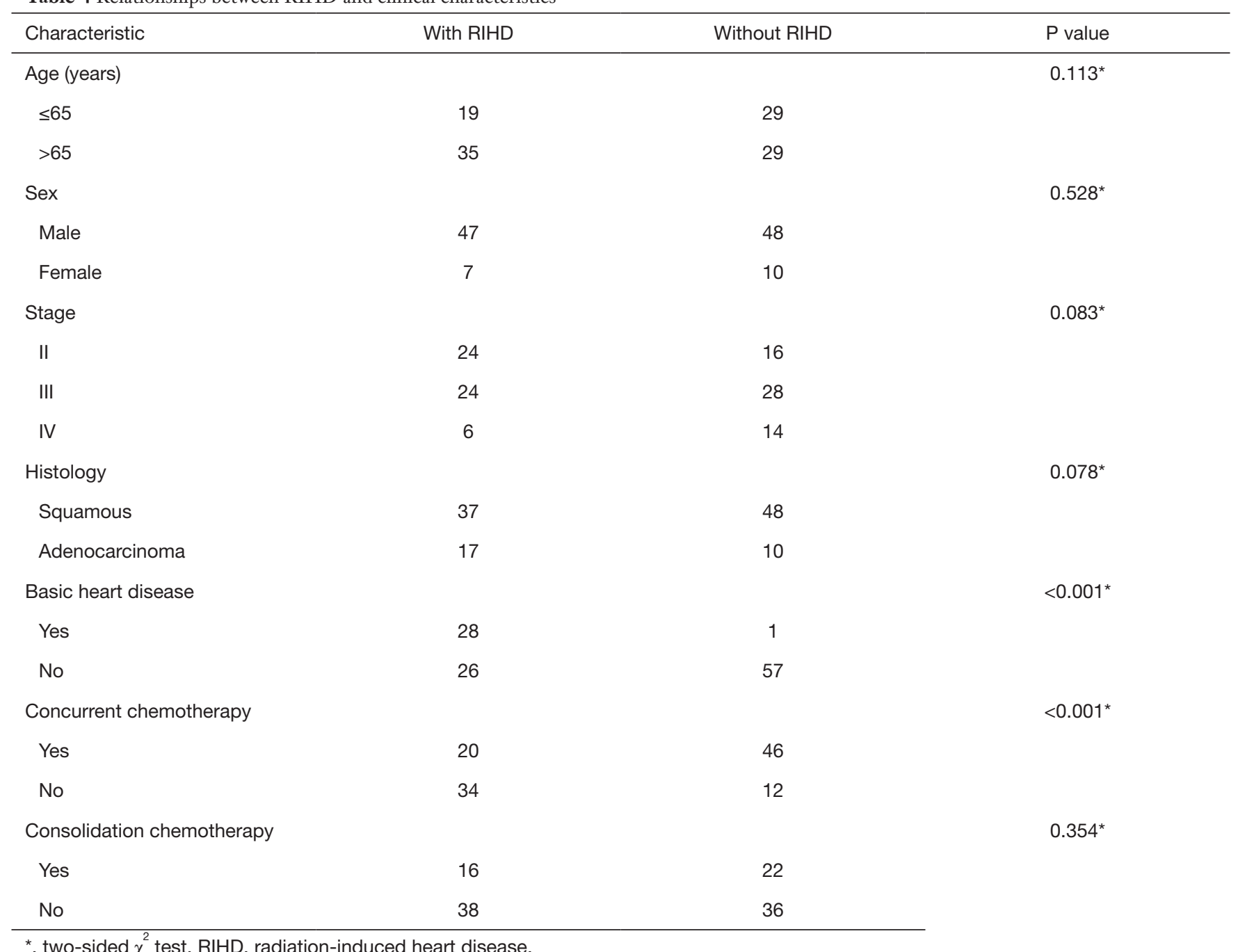

al. indicated that a minimized cardiac dose in RT planning was crucially important (13). Unfortunately, based on the available literature alone, it remains impossible to determine the most appropriate dose parameter, mainly due to the discrepancies in reported outcomes. Several articles examining the correlation between the dosevolume parameters of the heart and coronary disease have found heart V30 to be a significant predictor of radiationinduced pericardial effusion (9), while Tait $e t$ al. reported V40 as a possible predictive factor (20). In a study of 102 patients treated with concurrent chemoradiotherapy for locally advanced EC, Konski et al. predicted that the lowest significant cutoff values of V20, V30, and V40 were $70 \%$, $65 \%$, and $60 \%$, respectively (8). Similarly, in a study of long-term EC survivors, the thresholds for cardiac toxicity of V45, V50, and V55 were greater than $70 \%, 65 \%$, and $60 \%$, respectively (21). Compared with IMRT, proton beam therapy resulted in significantly lower mean heart dose (MHD) and heart V5, V10, V20, V30, and V40 as well as lower radiation exposure to the four chambers and four coronary arteries. Proton beam therapy results in significantly lower radiation exposure to the whole heart and cardiac substructures than IMRT (22).

These inconsistencies suggest that the variations in dose and fractionation, the definition of cardiac volumes with regard to radiation dosimetry, and the radiation technique used are important and cannot be ignored.

In our study, RIHD was more likely to occur in patients with basic heart disease, including $\mathrm{CAD}$, pericardial disease, and myocardial disease. Furthermore, of the chemotherapy 


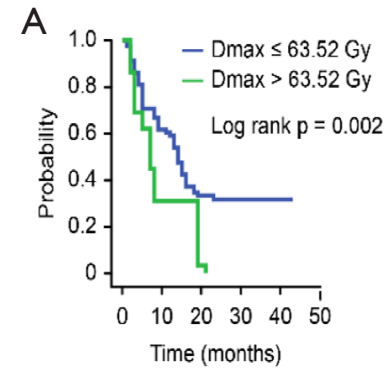

E
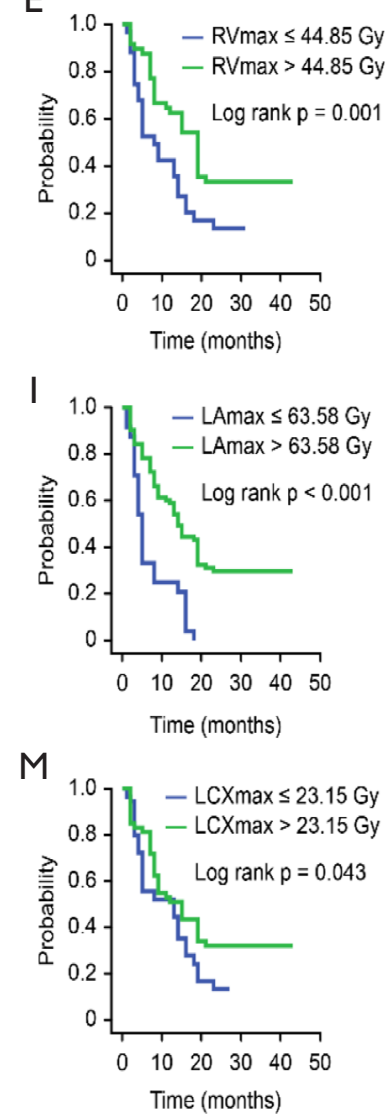

B

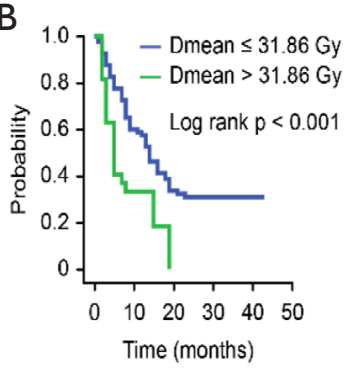

F
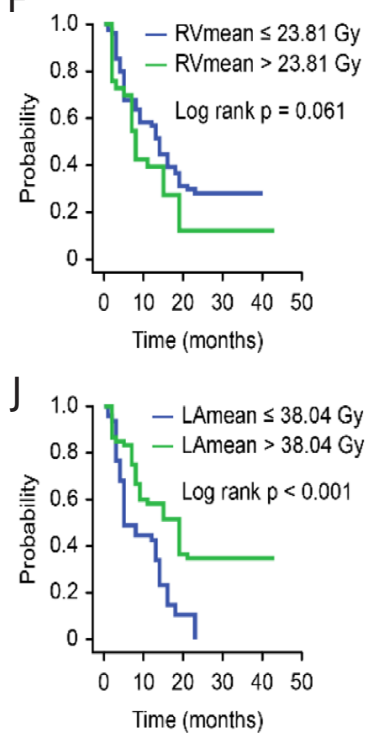

$\mathrm{N}$

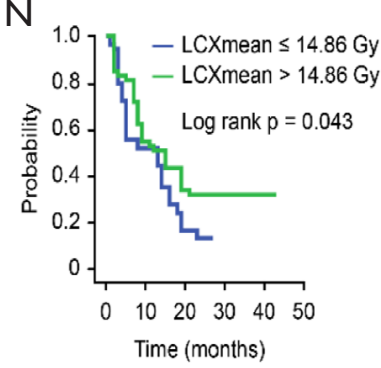

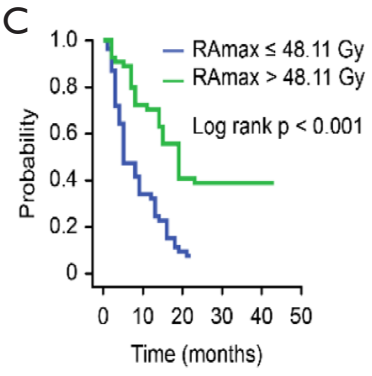

G
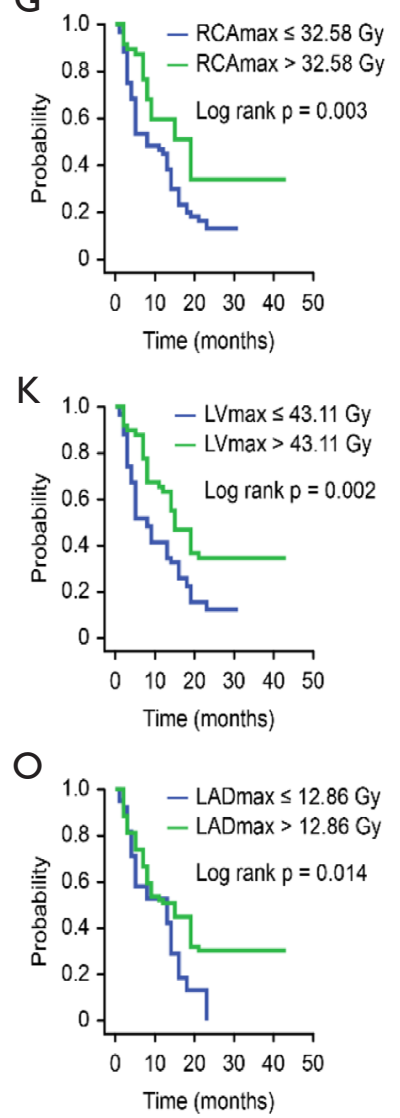

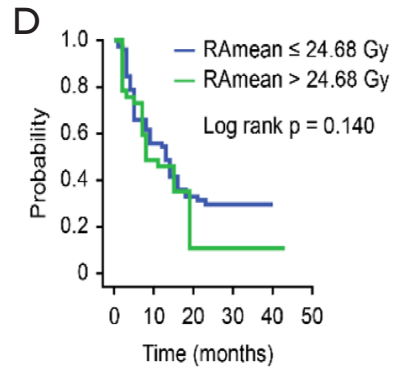

$\mathrm{H}$
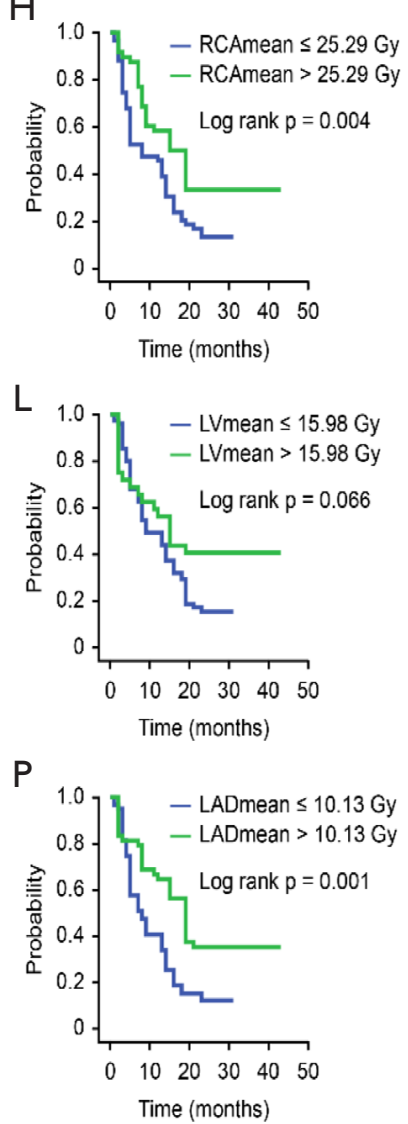

Figure 2 Kaplan-Meier curves for overall survival for various heart radiation dose parameters in advanced non-small cell lung cancer patients. (A) Dmax $\leq 63.52$ Gy (blue) vs. Dmax $>63.52$ Gy (green). (B) Dmean $\leq 31.86$ Gy (blue) vs. Dmean $>31.86$ Gy (green). (C) RAmax $\leq 48.11$ Gy (blue) vs. RAmax $>48.11$ Gy (green). (D) RAmean $\leq 24.68$ Gy (blue) vs. RAmean $>24.68$ Gy (green). (E) RVmax $\leq 44.85$ Gy (blue) vs. RVmax $>44.85$ Gy (green). (F) RVmean $\leq 23.81$ Gy (blue) vs. RVmean $>23.81$ Gy (green). (G) RCAmax $\leq 32.58$ Gy (blue) vs. RCAmax $>32.58$ Gy (green). (H) RCAmean $\leq 25.29$ Gy (blue) vs. RCAmean $>25.29$ Gy (green). (I) LAmax $\leq 63.58$ Gy (blue) $v s$.

regimens examined, concurrent chemoradiotherapy was associated with cardiac injury. This is in line with a study by Shapiro et al., who found chemoradiotherapy to be associated with an increased risk of cardiovascular adverse effects (23). Lee $e t$ al. also noted that concurrent chemotherapeutic agents, especially anthracyclines, were likely to potentiate a series of clinically notable myocardial diseases (17). Additionally, Stavrev et al. observed a higher incidence of pericardial effusion after chemoradiotherapy for EC, and Stavrev also found fraction size, bio-average and bio-maximum dose of heart are the risk factors for pericardial effusion $(24,25)$.

Although we obtained notable outcomes in our study, some limitations of this research should be mentioned. 
First, the records were derived from a retrospective cohort, and additional prospective studies are needed to confirm our findings. Second, the number of patients diagnosed with EC was relatively small, and larger, longer duration studies are required to validate these findings. Third, due to the small number of people with basic heart disease, subgroup analysis was not performed. The survival differences between patients with a previous history of heart disease and those with no history still needs further confirmation.

\section{Conclusions}

While RT plays an important role in the current treatment paradigm for EC, the use of RT in EC patients likely results in an increased risk of RIHD. Considering the cardiac toxicity, clinicians should monitor the doses received by the arteries of the heart. With regard to the probability of RIHD occurrence, other cardiac risk factors and the modality of chemotherapy should be subsequently monitored and managed. Limitation of the RT dose to the arteries of the heart should be carefully considered, and further work is necessary to minimize the risk of heart disease after RT for the treatment of EC.

\section{Acknowledgments}

Funding: This work was supported by grants from the National Natural Science Foundation of China (no. 81672974) and the Shandong Province Science Fund (no. SQ20170004).

\section{Footnote}

Reporting Checklist: The authors have completed the STROBE reporting checklist. Available at http://dx.doi. org/10.21037/atm-21-184

Data Sharing Statement: Available at http://dx.doi. org/10.21037/atm-21-184

Conflicts of Interest: All authors have completed the ICMJE uniform disclosure form (available at http://dx.doi. org/10.21037/atm-21-184). The authors have no conflicts of interest to declare.

Ethical Statement: The authors are accountable for all aspects of the work in ensuring that questions related to the accuracy or integrity of any part of the work are appropriately investigated and resolved. All patients who participated in this study provided written informed consent for publication. This study was approved by the Board and Ethical Committee of Qianfoshan Hospital and was conducted in accordance with the Declaration of Helsinki (as revised in 2013).

Open Access Statement: This is an Open Access article distributed in accordance with the Creative Commons Attribution-NonCommercial-NoDerivs 4.0 International License (CC BY-NC-ND 4.0), which permits the noncommercial replication and distribution of the article with the strict proviso that no changes or edits are made and the original work is properly cited (including links to both the formal publication through the relevant DOI and the license). See: https://creativecommons.org/licenses/by-nc-nd/4.0/.

\section{References}

1. McGuire S. World Cancer Report 2014. Geneva, Switzerland: World Health Organization, International Agency for Research on Cancer, WHO Press, 2015. Adv Nutr 2016;7:418-9.

2. Minsky BD, Pajak TF, Ginsberg RJ, et al. INT 0123 (Radiation Therapy Oncology Group 94-05) phase III trial of combined-modality therapy for esophageal cancer: high-dose versus standard-dose radiation therapy. J Clin Oncol 2002;20:1167-74.

3. Intensity Modulated Radiation Therapy Collaborative Working Group. Intensity-modulated radiotherapy: current status and issues of interest. Int J Radiat Oncol Biol Phys 2001;51:880-914.

4. Bouillon K, Haddy N, Delaloge S, et al. Long-term cardiovascular mortality after radiotherapy for breast cancer. J Am Coll Cardiol 2011;57:445-52.

5. Aleman BM, van den Belt-Dusebout AW, Klokman WJ, et al. Long-term cause-specific mortality of patients treated for Hodgkin's disease. J Clin Oncol 2003;21:3431-9.

6. Brusamolino E, Baio A, Orlandi E, et al. Long-term events in adult patients with clinical stage IA-IIA nonbulky Hodgkin's lymphoma treated with four cycles of doxorubicin, bleomycin, vinblastine, and dacarbazine and adjuvant radiotherapy: a single-institution 15 -year followup. Clin Cancer Res 2006;12:6487-93.

7. Hull MC, Morris CG, Pepine CJ, et al. Valvular dysfunction and carotid, subclavian, and coronary artery disease in survivors of hodgkin lymphoma treated with radiation therapy. JAMA 2003;290:2831-7. 
8. Konski A, Li T, Christensen M, et al. Symptomatic cardiac toxicity is predicted by dosimetric and patient factors rather than changes in $18 \mathrm{~F}-\mathrm{FDG}$ PET determination of myocardial activity after chemoradiotherapy for esophageal cancer. Radiother Oncol 2012;104:72-7.

9. Wei X, Liu HH, Tucker SL, et al. Risk factors for pericardial effusion in inoperable esophageal cancer patients treated with definitive chemoradiation therapy. Int J Radiat Oncol Biol Phys 2008;70:707-14.

10. Adams MJ, Lipshultz SE, Schwartz C, et al. Radiationassociated cardiovascular disease: manifestations and management. Seminars in radiation oncology. 2003;13:346-56.

11. Yeh ET, Tong AT, Lenihan DJ, et al. Cardiovascular complications of cancer therapy: diagnosis, pathogenesis, and management. Circulation 2004;109:3122-31.

12. Liu LK, Ouyang W, Zhao X,et al.Pathogenesis and Prevention of Radiation-induced Myocardial Fibrosis. Asian Pac J Cancer Prev2017;18:583-587.

13. Frandsen J, Boothe D, Gaffney DK, et al. Increased risk of death due to heart disease after radiotherapy for esophageal cancer. J Gastrointest Oncol 2015;6:516-23.

14. Lin SH, Wang L, Myles B, et al. Propensity score-based comparison of long-term outcomes with 3-dimensional conformal radiotherapy vs intensity-modulated radiotherapy for esophageal cancer. Int J Radiat Oncol Biol Phys 2012;84:1078-85.

15. Beukema JC, van Luijk P, Widder J, et al. Is cardiac toxicity a relevant issue in the radiation treatment of esophageal cancer? Radiother Oncol 2015;114:85-90.

16. Noël G, Mazeron JJ. Favourable and unfavourable effects on long-term survival of radiotherapy for early breast cancer: an overview of the randomised trials. Cancer Radiother 2001;5:92-4.

17. Lee PJ, Mallik R. Cardiovascular effects of radiation therapy: practical approach to radiation therapy-induced

Cite this article as: Zhang J, Lv Y, Chen F, Wang X, Zhang L, Zhang X. Doses of intensity-modulated radiotherapy and its association with cardiac disease in esophageal cancer patients. Ann Transl Med 2021;9(2):166. doi: 10.21037/atm-21184 heart disease. Cardiol Rev 2005;13:80-6.

18. Lorenzen EL, Taylor CW, Maraldo M, et al. Interobserver variation in delineation of the heart and left anterior descending coronary artery in radiotherapy for breast cancer: a multi-centre study from Denmark and the UK. Radiother Oncol 2013;108:254-8.

19. Gyenes G, Rutqvist LE, Liedberg A, et al. Longterm cardiac morbidity and mortality in a randomized trial of pre- and postoperative radiation therapy versus surgery alone in primary breast cancer. Radiother Oncol 1998;48:185-90.

20. Tait LM, Meyer JE, McSpadden E, et al. Women at increased risk for cardiac toxicity following chemoradiation therapy for esophageal carcinoma. Pract Radiat Oncol 2013;3:e149-55.

21. Ogino I, Watanabe S, Iwahashi N, et al. Symptomatic radiation-induced cardiac disease in long-term survivors of esophageal cancer. Strahlenther Onkol 2016;192:359-67.

22. Kole TP, Aghayere O, Kwah J, et al. Dosimetric comparison to the heart and cardiac substructure in a large cohort of esophageal cancer patients treated with proton beam therapy or Intensity-modulated radiation therapy. Radiother Oncol2017;125:48-54.

23. Shapiro CL, Hardenbergh PH, Gelman R, et al. Cardiac effects of adjuvant doxorubicin and radiation therapy in breast cancer patients. J Clin Oncol 1998;16:3493-501.

24. Stavrev P, Stavrev N. Fraction size and dose parameters related to the incidence of pericardial effusions: regarding Martel et al. IJROBP 40(1):155-161; 1998. Int J Radiat Oncol Biol Phys 2000;48:611-3.

25. Ishikura S, Nihei K, Ohtsu A, et al. Long-term toxicity after definitive chemoradiotherapy for squamous cell carcinoma of the thoracic esophagus. J Clin Oncol 2003;21:2697-702.

(English Language Editor: J. Gray) 